\title{
Multiple Plane Phase Retrieval Based On Inverse Regularized Imaging and Discrete Diffraction Transform
}

\author{
Artem Migukin, Vladimir Katkovnik, and Jaakko Astola \\ Department of Signal Processing, Tampere University of Technology (TUT), \\ Korkeakoulunkatu 10, P.O.Box 553, Fi-33720, Tampere, Finland \\ artem.migukin@tut.fi,_vladimir.katkovnik@tut.fi, jaakko.astola@tut.fi
}

\begin{abstract}
The phase retrieval is formulated as an inverse problem, where the forward propagation is defined by Discrete Diffraction Transform (DDT) [1], [2]. This propagation model is precise and aliasing free for pixelwise invariant (pixelated) wave field distributions in the sensor and object planes. Because of finite size of sensors DDT can be illconditioned and the regularization is an important component of the inverse. The proposed algorithm is designed for multiple plane observations and can be treated as a generalization of the Gerchberg-Saxton iterative algorithm. The proposed algorithm is studied by numerical experiments produced for phase and amplitude modulated object distributions. Comparison versus the conventional forward propagation models such as the angular spectrum decomposition and the convolutional model used in the algorithm of the same structure shows a clear advantage of $D D T$ enabling better accuracy and better imaging.
\end{abstract}

Keywords: phase retrieval, digital image processing algorithms, computer generated holograms

PACS: 42.30.Rx, 07.05.Pj, 42.40.Jv

\section{INTRODUCTION}

The phase of radiation scattered from an object carries important information about an object surface and its properties. Phase measurements are exploited in many fields in materials and biological sciences. Because only the magnitude (intensity) of radiation can be measured directly the problem of phase reconstruction from intensity measurements appears. Solutions fall into two large categories: interferometric (holographic) with a reference beam and non-interferometric (phase-retrieval), where a reference beam is not required. The latter solution is substantially simpler and, what is important, much more robust with respect to disturbances.

The method proposed by Gerchberg and Saxton [3] is the first popular beam-propagation-based phase reconstruction method. The idea is that the phases missing in observations are recovered iteratively applying the magnitude constraints in object and sensor planes. This technique has been studied, modified and developed in a flow of publications (e.g. [4], [5], [6]). In this paper we consider a single and multiple plane observation scenarios where the magnitudes of the wave field are measured in the planes parallel to the object plane. It is assumed that the wave field is generated by radiation from the object plane. The reconstruction of the phases missed in observations is produced through the object plane distribution considered as the only unknown variable.

The proposed iterative algorithm is a generalization of the Gerchberg and Saxton algorithm for multiple plane observations and inverse imaging techniques. In this work we are restricted to numerical experiments and the forward wave filed propagation modeling based on the discrete diffraction transform (DDT) developed in two complementary forms: in the frequency (Fourier) domain $(F-D D T)[1]$ and in the spatial domain as the matrix discrete diffraction transform $(M-D D T)$ [2]. By its very nature this transform enables the perfect forward propagation modeling for the pixelated wave field. The "pixelated" assumes that the wave field distributions are pixelwise invariant in the object and sensor planes. This assumption is natural for all sorts of digital sensors and used as a pixelwise approximation for the object plane. In numerical modeling we always deal with the pixelated objects and in this way we can claim that at least for numerical experiment this wave field modeling is accurate.

The contribution of this paper concerns a demonstration that the developed algorithm enables a better accuracy in comparison with the standard ones and better visualization. In this comparison we study as valuable alternatives the algorithms using the angular spectrum decomposition $(A S D)$ and discrete convolution $(D C)$ as models for the 
forward propagation modeling. We consider the reconstruction of the object wave fields with amplitude or phase modulations. It is shown that the spatially adaptive regularization used in the inverse imaging results in the further improvement of the algorithm performance.

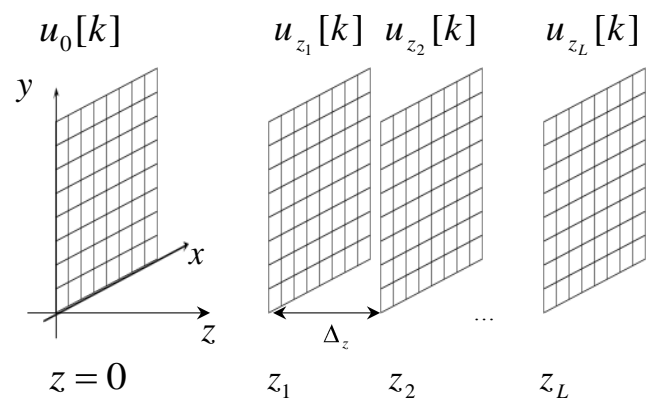

FIGURE 1. Multiple plane phase (wave field) reconstruction: $u_{0}$ is an object plane, $u_{z_{l}}$ are measurement planes, $l=1, \ldots, L$.

\section{OBSERVATION MODELS}

Let $u_{0}(x)$ and $u_{z_{l}}(x), x \in R^{2}, l=1, \ldots, L$, denote the complex-valued wave field distributions in the object and sensor planes respectively. $z_{l}$ indicates a distance between the parallel object and $l$-th planes, and $L$ is a number of the observation planes (sensors) (see Fig. 1). The pixelated modeling means that the continuous arguments $x$ are replaced by integer ones with the following replacements of the continuous distributions by their discrete models: $u_{0}(x) \rightarrow u_{0}[k], u_{z_{l}}(x) \rightarrow u_{z_{l}}[k], k \in Z^{2}$. Thus, the argument $k$ is a two dimensional vector with integer components, i.e. belongs to the set $Z^{2}$. For a finite size square image it means that $k=\left(k_{1}, k_{2}\right), 0 \leq k_{1}, k_{2} \leq N-1$. In $D D T$ the values $u_{0}[k]$, $u_{z_{l}}[k]$ are defined as the mean values of the corresponding continuous variables with the means calculated over the square or rectangular pixels. The link between these $u_{0}[k]$ and $u_{z l}[k]$ is accurate for the pixelwise object distribution and the discrete sensor. In the frequency domain this link can be given in the form [1]

$$
\tilde{U}_{z_{l}}[f]=\tilde{A}_{z_{l}, z_{0}}[f] \cdot \tilde{U}_{0}[f],
$$

where $\tilde{U}_{0}[f]$ and $\tilde{U}_{z_{l}}[f]$ are calculated as the fast Fourier transform (FFT) of $\tilde{u}_{0}[k]$ and $\tilde{u}_{z_{l}}[k], f \in Z^{2}$ and the transfer function $\tilde{A}_{z_{p} z_{0}}[f]$ is $F F T$ of the pixelwise averaged values of the kernel of the Rayleigh-Sommerfeld integral. The calculation of $\tilde{A}_{z_{p} z_{0}}[f]$ in [1] is produced for the Fresnel approximation of this kernel.

In what follows $\tilde{u}_{0}[k]$ and $\tilde{u}_{z l}[k]$ are extended double size versions of $u_{0}[k]$ and $u_{z_{l}}[k]$ zero padded to the double size of the images, and all frequency domain operations are produced for the double size images. There are two reasons for these double size operations. First, we need this double size in order to the $D D T$ forward propagation be accurate. $u_{z_{l}}[k]$ is calculated from the double size inverse $F F T\left(\tilde{u}_{z_{l}}[k]=F F T^{-1}\left\{\tilde{U}_{z_{l}}[f]\right\}\right)$ by taking the middle part of this double size image. Remind that these sort of double size calculations are typical for accurate convolutional techniques (e.g. [7]). Second, the extra area of images appeared due to zero padding is essential for the recursive inverse performed in the frequency domain [1]. Concerning $f$ in (1) note, that it is a vector $f=\left(f_{1}, f_{2}\right),-N \leq f_{1}, f_{2} \leq N-1$. The integer 2D regular grid for $f$ is circular-shifted in such a way that the origin point $f_{1}=0, f_{2}=0$ is in the left upper corner of the grid.

The frequency models similar to (1) can be used for all forward propagation models. Here we mention only two of them used in our numerical experiments. The transfer function for $A S D$ is calculated analytically as follows [8]:

$$
A S D_{z_{l}, z_{0}}[f]=\exp \left[j 2 \pi z_{l} / \lambda \cdot \sqrt{1-\lambda^{2}\|f\|^{2} /(\Delta N)^{2}}\right], \lambda^{2} /(\Delta N)^{2}<1
$$

The transfer function for the discrete convolutional $(D C)$ model is calculated as [7]:

$$
D C_{z_{l}, z_{0}}[f]=F F T\left\{g_{z_{l}}[k \Delta]\right\}, g_{z_{l}}(x)=z_{l} / r_{l}^{2} \cdot\left[1 /(j \lambda)+1 /\left(2 \pi r_{l}\right)\right] \cdot \exp \left(j 2 \pi r_{l} / \lambda\right), r_{l}=\sqrt{z_{l}^{2}+\|x\|^{2}}
$$

In these formulas $\lambda$ is the wavelength, and $\Delta$ is a parameter of the pixel size (we assume that the pixels are square $\Delta \times \Delta$ ). The transfer functions (2)-(3) are exploited for calculations of the spectrum in the sensor planes based on the formula (1). These calculations can be produced for double and single size images. 


\section{PHASE RETRIEVAL ALGORITHM}

Let us assume for a moment that the complex-valued $u_{z_{l}}$ are known. Then the reconstruction of $u_{0}$ can be produced in the frequency domain according to the following optimization formulation:

$$
\tilde{U}_{0}[f]=\arg \min _{\tilde{U}_{0}[f]} J, J=\sum_{l=1}^{L}\left\|\widetilde{U}_{z_{l}}[f]-\tilde{A}_{z_{l}, z_{0}}[f] \cdot \tilde{U}_{0}[f]\right\|_{2}^{2}+\alpha^{2}\left\|\widetilde{U}_{0}[f]\right\|_{2}^{2},
$$

where the Euclidean norm means $\left\|\tilde{U}_{0}[f]\right\|_{2}{ }^{2}=\Sigma_{f}\left|\tilde{U}_{0}[f]\right|^{2}$ and $\alpha^{2}$ is a regularization parameter.

The routine calculations give the estimate of $\tilde{U}_{0}[f]$ in the form

$$
\tilde{U}_{0}[f]=\left(\sum_{l=1}^{L} \tilde{A}_{z_{l}, z_{0}}^{*}[f] \cdot \tilde{U}_{z_{l}}[f]\right) /\left(\sum_{l=1}^{L}\left|\tilde{A}_{z_{l}, z_{0}}[f]\right|^{2}+\alpha^{2}\right)
$$

where ${ }^{\prime * \prime}$ stands for a complex-conjugate variable. The criterion in (4) corresponds to the standard Tikhonov's quadratic regularization of the ill-conditioned inverse problems [9]. We will use the formula (5) in order to derive the algorithm for the case, when only magnitude data are available instead of the complex valued one.

Let the observations in the sensor planes be given as

$$
o_{z_{l}}[k]=\left|u_{z_{l}}[k]\right|+\varepsilon_{l}[k],
$$

where $\varepsilon_{l}[k]$ are measurement noises.

Substitute these noisy magnitude observations in (5) instead of $u_{z_{l}}$, then we arrive to the following expressions:

$$
\begin{aligned}
& \tilde{U}_{0}[f]=\left(\sum_{l=1}^{L} \tilde{A}_{z_{l}, z_{0}}^{*}[f] \cdot F F T\left\{\tilde{v}_{l}[k]\right\}\right) /\left(\sum_{l=1}^{L}\left|\tilde{A}_{z_{l}, z_{0}}[f]\right|^{2}+\alpha^{2}\right), \\
& \tilde{v}_{l}[k]=\left[\tilde{o}_{z_{l}}[k] \cdot I[k]+\left|\tilde{u}_{z_{l}}[k]\right| \cdot(1-I[k])\right] \cdot \exp \left(j \tilde{\phi}_{l}\right), \\
& \tilde{\phi}_{l}=\operatorname{angle}\left(\tilde{u}_{z_{l}}\right), \tilde{u}_{z_{l}}=F F T^{-1}\left\{\tilde{A}_{z_{l}, z_{0}}[f] \cdot \tilde{U}_{0}[f]\right\} .
\end{aligned}
$$

Here $I[k]$ is the indicator of the image area, i.e. $I[k]=1$ for the image area and equal to 0 otherwise. Then the formula $\tilde{o}_{z_{l}}[k] \cdot I[k]+\left|\tilde{u}_{z_{l}}[k]\right| \cdot(1-I[k])$ replaces the magnitude of $u_{z_{l}}[k]$ by the observations $o_{z_{l}}[k]$ in the middle part of the double size image and preserves the magnitudes of $\tilde{u}_{z_{l}}[k]$ calculated for the outside of this middle part. The formulas in (7) are equations with respect to unknown $u_{0}[k]$. These equations are nonlinear because the phases $\tilde{\phi}_{l}$ in the observation planes depend on $u_{0}[k]$.

The following iterative procedure is natural for these equations and defines the proposed iterative algorithm:

$$
\begin{aligned}
& \tilde{U}_{0}^{(t+1)}[f]=\left(\sum_{l=1}^{L} \tilde{A}_{z_{l}, z_{0}}^{*}[f] \cdot F F T\left\{\tilde{v}_{l}^{(t)}[k]\right\}\right) /\left(\sum_{l=1}^{L}\left|\tilde{A}_{z_{l}, z_{0}}[f]\right|^{2}+\alpha^{2}\right), \\
& \tilde{v}_{l}^{(t)}[k]=\left[\tilde{o}_{z_{l}}[k] \cdot I[k]+\left|\tilde{u}_{z_{l}}^{(t)}[k]\right| \cdot(1-I[k])\right] \cdot \exp \left(j \tilde{\phi}_{l}^{(t)}\right), \\
& \tilde{\phi}_{l}^{(t)}=\operatorname{angle}\left(\tilde{u}_{z_{l}}^{(t)}\right), \tilde{u}_{z_{l}}^{(t)}=F F T^{-1}\left\{\tilde{A}_{z_{l}, z_{0}}[f] \cdot \tilde{U}_{0}^{(t)}[f]\right\}, t=0,1, \ldots
\end{aligned}
$$

We name this algorithm Multiple Plane Frequency DDT $(M F-D D T)$. This iterative procedure can be used also with the $A S D$ and $D C$ frequency domain models (transfer functions (2) and (3)). The calculations become simpler because in this case $\left|\tilde{A}_{z_{p} z_{0}}[f]\right|^{2}=1, \tilde{A}_{z_{p} z_{0}}{ }^{*}[f]=\tilde{A}_{-z_{p} z_{0}}[f]$, and we do not need regularization. We will refer to the algorithms generated from (8) as to the $A S D$ or $D C$ algorithms depending on which forward propagation model is used.

It is not difficult to realize that the algorithm (8) can be treated as a generalization of the Gerchberg-Saxton algorithm for multiple observation planes and the $D D T$ forward propagation requiring the regularized inverse for the backward propagation.

The single-beam multiple-intensity phase reconstruction (SBMIR) technique using only the intensity measurements of a volume speckle field and wave propagation equation has been proposed and studied in [10], [11]. The phase distributions for the observation planes are reconstructed successively in circular iterations from one observation plane to the following one starting from the first observation plane. The algorithm (8) is different from SBMIR in principle because the observations from all planes are processed simultaneously with estimation of $u_{0}$ as the only unknown variable. The simulation confirms the advantage of this parallel processing of all observations.

A further development of the proposed approach is produced by using a varying spatially adaptive regularization instead of the simple Tikhonov`s one. It is shown in [12] that this sort of regularization can be implemented as 
spatially adaptive filtering. In the developed modification of the algorithm (8) the phase and the magnitude of each iterative estimate $\tilde{u}_{0}{ }^{(t)}[k]$ are subjects of the special filtering. For this filtering we use the powerful adaptive BM3D algorithm [13]. Simulation demonstrates an essential improvement of these phase and magnitude estimates visually and numerically.

\section{NUMERICAL EXPERIMENTS}

We tested our algorithm in multiple experiments with amplitude (AM) or phase (PM) modulated object distributions. The images are square $N \times N, N=256$ with square pixels $\Delta \times \Delta, \Delta=6.7 \mu \mathrm{m}$, the wavelength $\lambda=632.8 \mathrm{~nm}$. The "in-focus" distance is calculated as $z_{f}=N \Delta^{2} / \lambda=18.16 \mathrm{~mm}$. It is assumed that the additive noise in (6) is zeromean Gaussian with $\sigma=0.01$. The number of measurement planes is varying from $L=1$ to $L=20$. The number of iterations is fixed to 100 . As an image used for modulation of the object wave field distribution we use the standard test-image lena. The distances between the measurement planes are constant and equal to $\Delta_{\mathrm{z}}=0.5 \mathrm{~mm}$. $z_{1}$ is the distance from the object to the first measurement plane (see Fig. 1).

It is appeared that the $D C$ algorithm fails for $z_{l}<z_{f}$ due to strong aliasing effects and nearly equivalent to the $A S D$ algorithm for $z_{1} \geq z_{f}$. In what follows we show the results only for $M F-D D T$ and $A S D$ algorithm and drop the results for the $D C$ algorithm.
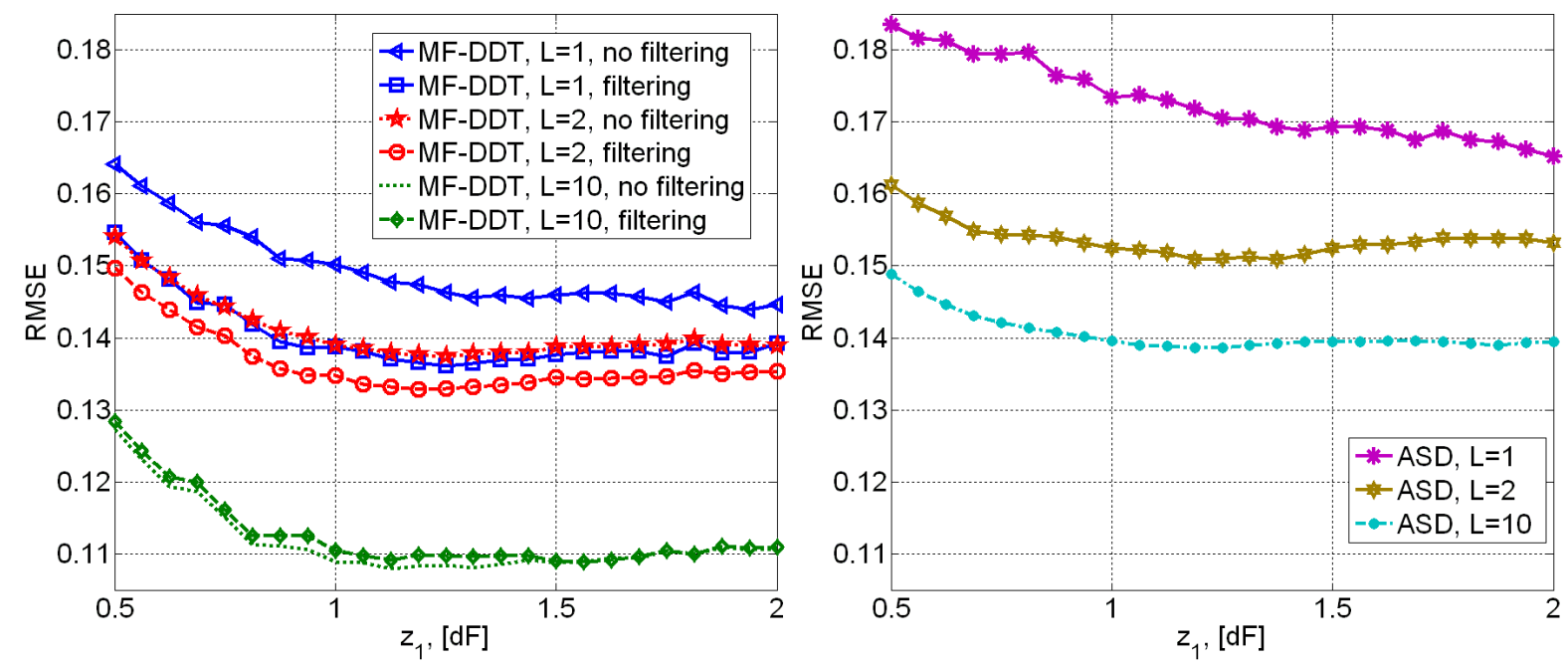

FIGURE 2. The accuracy (RMSE) of the object phase reconstruction by the $M F-D D T$ and ASD algorithms versus the distance $z_{1}$. This distance is given as a ratio to the "in-focus" distance $z_{f}$.

The accuracy of the phase reconstructions in the object plane is shown in Fig. 2 for different distances $z_{1}$. The accuracy of $M F-D D T$ is always better than that for the $A S D$ algorithm with the relative improvement in $R M S E$ values about $30 \div 50 \%$. The adaptive regularization embedded in the $M F-D D T$ algorithm (BM3D filtering) improves the accuracy and visual perception of images.

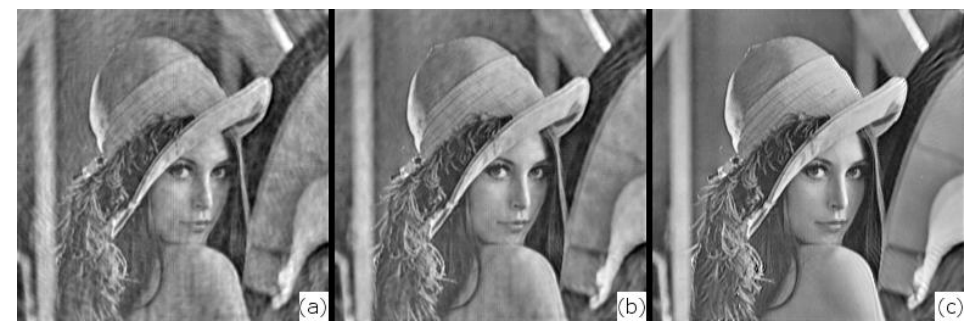

FIGURE 3. The phase reconstruction by the $M F-D D T$ algorithm $\left(z_{l}=1.5 \cdot z_{f}\right):$ (a) $L=1, P S N R=15.9 \mathrm{~dB}$; (b) $L=2, P S N R=16.3 \mathrm{~dB}$; (c) $L=10, P S N R=16.8 \mathrm{~dB}$. 


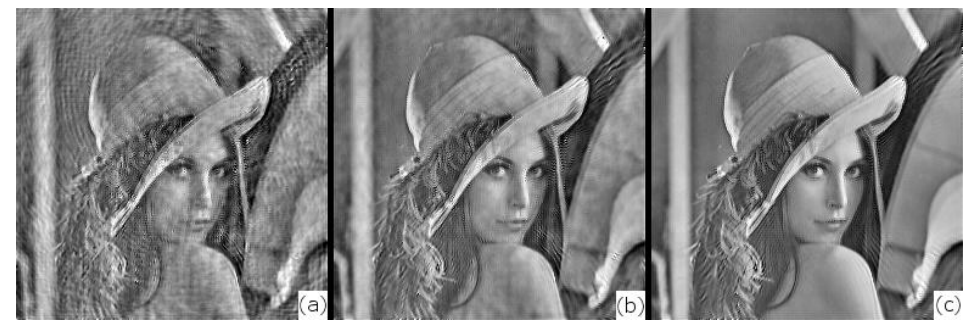

FIGURE 4. The phase reconstruction by the $A S D$ algorithm $\left(z_{1}=1.5 \cdot z_{f}\right)$ : (a) $L=1, P S N R=14.6 \mathrm{~dB}$; (b) $L=2, P S N R=15.5 \mathrm{~dB}$; (c) $L=10, P S N R=16.3 \mathrm{~dB}$.

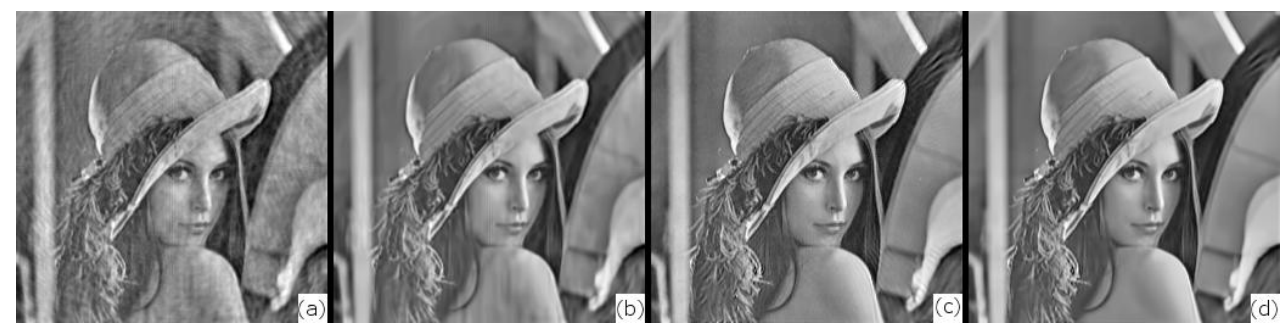

FIGURE 5. The filtering effects in the phase reconstruction by the $M F-D D T$ algorithm $\left(z_{1}=1.5 \cdot z_{f}\right):$ (a) $L=1, P S N R=15.9 \mathrm{~dB}$ (no filtering); (b) $L=1, P S N R=16.4 \mathrm{~dB}$ (with filtering); (c) $L=10, P S N R=16.8 \mathrm{~dB}$ (no filtering); (d) $L=10, P S N R=17 \mathrm{~dB}$ (with filtering).

Examples of images obtained from the phase reconstruction by $M F-D D T$ and $A S D$ algorithms are presented in Fig. 3 and Fig. 4 respectively. The image quality is characterized by PSNR values calculated in $\mathrm{dB}$ as $20 \log _{10}\left(\max \left|u_{o}\right| / R M S E\right)$. For the $M F-D D T$ algorithm the enhancement in the image quality is quite clear visually and numerically with $P S N R$ improvement equal to about $0.4 \mathrm{~dB}$ for $L=2$ as compared with $L=1$. $L=10$ gives a further improvement in $P S N R$ of about $0.5 \mathrm{~dB}$ as compared with $L=2$. For the $A S D$ algorithm the improvements in $P S N R$ values even more essential for larger values of $L$ as it can be seen in Fig. 4.

The filtering (adaptive regularization) in the $M F-D D T$ algorithm is valuable for imaging as it is demonstrated in Fig. 5 for $L=1$ and $L=10$.

A larger number of the observation planes $L$ results in a better accuracy for the $M F-D D T$ algorithm, and this improvement is very valuable for $L=2$. Higher values of $L$ monotonically improve the accuracy but not so efficiently. The dependences of RMSE on the number of the observation planes $L$ for $M F-D D T$ algorithm with filtering and without filtering are shown in Fig. 6a. All results in this figure are given for the observations obtained by the $D D T$ forward propagation modeling. For comparison we show also the accuracy achieved by the $A S D$ algorithm. We can see that the accuracy of the $M F-D D T$ algorithm is much better for all numbers of the observation planes from $L=1$ up to $L=20$. This difference in the performance of the algorithms is defined by the fact that the $M F$ $D D T$ algorithm is a more appropriate fit (than the $A S D$ algorithm) to the forward $D D T$ propagation model used to generate the observations. In this way $M F-D D T$ is able to give better results. The RMSE curves in Fig. 6a show that this potential is well realized by the $M F-D D T$ algorithm.
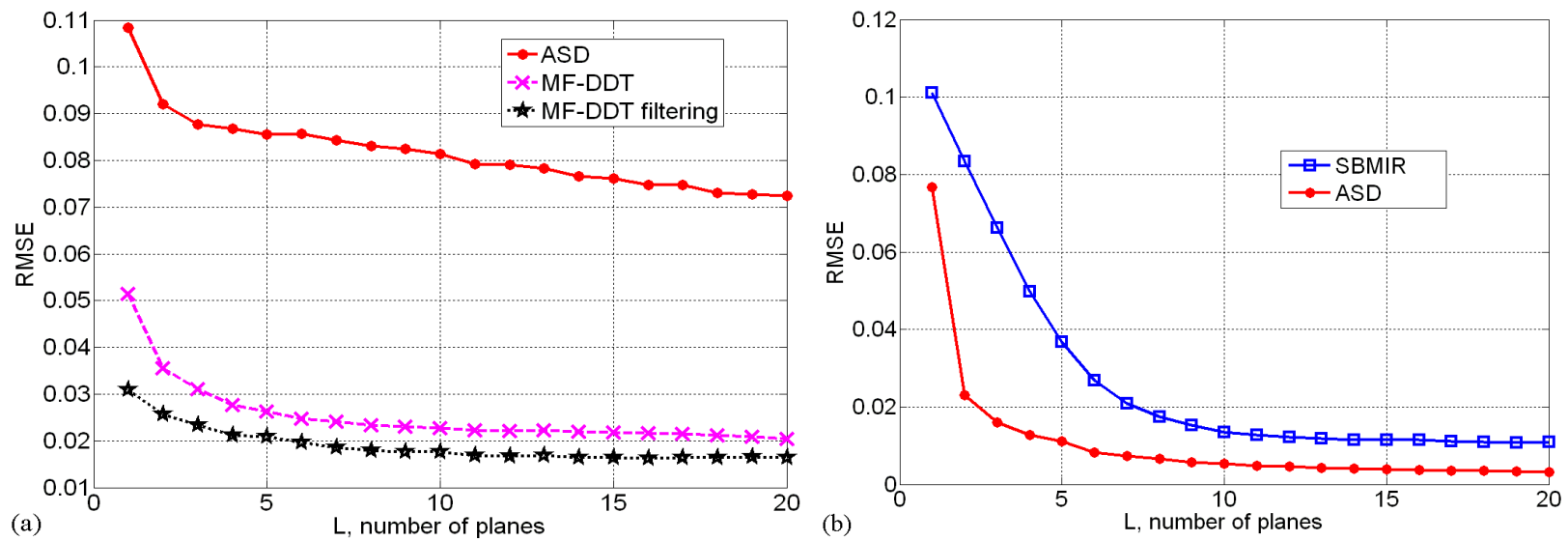

FIGURE 6. The object magnitude reconstruction, RMSE accuracy of the $M F-D D T$, ASD and SBMIR algorithms versus the number of planes $L, z_{1}=1.5 \cdot z_{f}$. Observed data are generated according to the forward propagation models: (a) $D D T$, (b) ASD. 
The only difference between the SBMIR and ASD algorithms is in their structure: plane-to-plane phase reconstruction in SBMIR and parallel plane processing in the $A S D$ as it is discussed in Section "Phase retrieval algorithm". In Fig.6b we produce a comparison of these algorithms. This comparison is done for observations generated by the ASD forward propagation model. Remind that the wave field propagation in the SBMIR algorithm is implemented according to the considered $A S D$ model [11]. In this case the wave field propagation used in the both algorithms accurately corresponds to the observations generated by the $A S D$ model. Thus, we obtain the scenario for an accurate comparison of two different ideas: the parallel versus successive plane-by-plane data processing. The RMSE curves in Fig.6b show uniformly smaller RMSE values for the ASD algorithm. We consider it as an evidence in favor of the parallel processing with respect to the successive one. For the data generated according to the forward $D D T$ propagation model the SBMIR algorithm demonstrates the performance which is similar but a bit worse than that shown in Fig.6a for the $A S D$ algorithm.

Once more note that for the pixelated object and sensor planes the forward DDT propagation modeling is accurate. It is the main reason behind evaluation of the algorithms on the data generated according to this model.

\section{CONCLUSIONS}

In this work we present a multiple plane phase retrieval algorithm based on simultaneous (parallel) data processing and $D D T$ as the forward propagation model. Both things are essential to explain the advance performance of the proposed algorithm. Multiple experiments have been produced in order to study the algorithm behavior for different test images and for reconstruction of phase/magnitude/complex-valued object distributions. Only a small part of these results are shown in the paper. Our further work concerns the adaptive selection of the regularization parameter as well as a tuning of the adaptive filtering, in particular for noisy data. We wish to note that the developed approach is quite flexible. In particular, it can be applied for wave field reconstruction from multiple wavelength data.

\section{ACKNOWLEDGMENTS}

This research is supported by the Academy of Finland, project No. 213462 (Finnish Centre of Excellence program 2006 - 2011), and the post graduate work of Artem Migukin is funded by the Tampere Graduate School in Information Science and Engineering (TISE).

\section{REFERENCES}

1. V. Katkovnik, J. Astola, and K. Egiazarian, Appl. Opt. 47, 3481-3493 (2008).

2. V. Katkovnik, A. Migukin, and J. Astola, Appl. Opt. 48, 3407-3423 (2009).

3. R. W. Gerchberg and W. O. Saxton, Optik 35, 227-246 (1972).

4. J. R. Fienup, Optics Lett. 3, 27-29 (1978).

5. J. R. Fienup, Appl. Opt. 21, 2758-2769 (1982).

6. M. Guizar-Sicairos and J. R. Fienup, Opt. Express 16, 7264-7278 (2008).

7. F. Shen and A. Wang, Appl. Opt. 45, 1102-1110 (2006).

8. J. W. Goodman, Introduction to Fourier Optics, New York: McGraw-Hill Inc, Second Edition, 1996.

9. A.N. Tikhonov and V.Y. Arsenin. Solution of ill-posed problems, New York: Wiley, 1977.

10. G. Pedrini, W. Osten, and Y. Zhang, Opt. Lett. 30, 833-835 (2005).

11. P. Almoro, G. Pedrini and W. Osten, Appl. Opt. 45 8596-8605 (2006).

12. K. Dabov, A. Foi, and K. Egiazarian, Proc. SPIE Electronic Imaging '08, no. 6812-07, San Jose, California, USA, 2008

13. K. Dabov, A. Foi, V. Katkovnik, and K. Egiazarian, Proc. SPIE Electronic Imaging '06, no. 6064A-30, San Jose, California, USA, 2006 